\title{
Risk of herpes zoster among patients with chronic obstructive pulmonary disease: a population-based study
}

\author{
Ya-Wen Yang MD MS, Yi-Hua Chen PhD, Kuo-Hsien Wang MD MS, Chen-Yi Wang MD, Hui-Wen Lin PhD
}

\begin{abstract}
- ABSTRACT
Background: Systemic inflammation and dysregulated immune function in chronic obstructive pulmonary disease (COPD) is hypothesized to predispose patients to development of herpes zoster. However, the risk of herpes zoster among patients with COPD is undocumented. We therefore aimed to investigate the risk of herpes zoster among patients with COPD.

Methods: We conducted a cohort study using data from the Taiwan Longitudinal Health Insurance Database. We performed Cox regressions to compare the hazard ratio (HR) of herpes zoster in the COPD cohort and in an age- and sex-matched comparison cohort. We divided the patients with COPD into three groups according to use of steroid medications and performed a further analysis to examine the risk of herpes zoster.
\end{abstract}

Results: The study included 8486 patients with COPD and 33944 matched control patients. After adjustment for potential confounding factors, patients with COPD were more likely to have incidents of herpes zoster (adjusted HR $1.68,95 \%$ confidence interval [Cl] 1.45-1.95). When compared with the comparison cohort, the adjusted HR of herpes zoster was 1.67 (95\% Cl 1.43-1.96) for patients with COPD not taking steroid medications. The adjusted HR of herpes zoster was slightly greater for patients with COPD using inhaled corticosteroids only (adjusted HR 2.09, 95\% Cl 1.38-3.16) and was greatest for patients with COPD using oral steroids (adjusted HR 3.00, 95\% Cl 2.40-3.75).

Interpretation: Patients with COPD were at increased risk of herpes zoster relative to the general population. The relative risk of herpes zoster was greatest for patients with COPD using oral steroids.
Competing interests: None declared.

This article has been peer reviewed.

Correspondence to:

Dr. Hui-Wen Lin,

linhw@tmu.edu.tw

CMAJ 2011. DOI:10.1503 /cmaj.101137
$\mathrm{H}$ erpes zoster is caused by a reactivation of latent varicella-zoster virus residing in sensory ganglia after an earlier episode of varicella. ${ }^{1}$ Herpes zoster is characterized by a painful vesicular dermatomal rash. It is commonly complicated with chronic pain (postherpetic neuralgia), resulting in reduced quality of life and functional disability to a degree comparable to that experienced by patients with congestive heart failure, diabetes mellitus and major depression. ${ }^{1.2}$ Patients with herpes zoster experience more substantial role limitations resulting from emotional and physical problems than do patients with congestive heart failure or diabetes. ${ }^{3}$ Pain scores for postherpetic neuralgia have been shown to be as high as those for chronic pain from osteoarthritis and rheumatoid arthritis. ${ }^{3}$ Although aging is the most well-known risk factor for herpes zoster, people with diseases associated with impaired immunity, such as malignancy, HIV infection, diabetes and rheumatic diseases, are also at higher risk for herpes zoster. ${ }^{4.5}$
Chronic obstructive pulmonary disease (COPD) is characterized by progressive airflow limitation that is associated with an abnormal inflammatory response by the small airways and alveoli to inhaled particles and pollutants. ${ }^{6}$ Disruption of local defence systems (e.g., damage to the innate immune system, impaired mucociliary clearance) predispose patients with COPD to respiratory tract infections. Each infection can cause exacerbation of COPD and further deterioration of lung function, which in turn increase predisposition to infection. ${ }^{7.8}$

There is increasing evidence that COPD is an autoimmune disease, with chronic systemic inflammation involving more than just the airways and lungs. ${ }^{6}$ Given that various immune-mediated diseases (e.g., rheumatoid arthritis, inflammatory bowel disease) have been reported to be associated with an increased risk of herpes zoster, ${ }^{4,910}$ it is reasonable to hypothesize that the immune dysregulation found in COPD may put patients at higher risk of developing herpes zoster. In addition, inhaled or systemic corticosteroids used for management of 
COPD can increase susceptibility to herpes zoster by suppressing normal immune function. ${ }^{11}$ However, data are limited regarding the risk of herpes zoster among patients with COPD.

The goal of our study was to investigate whether patients with COPD have a higher incidence of herpes zoster than the general population. In addition, we aimed to examine the risk for herpes zoster with and without steroid therapy among patients with COPD relative to the general population.

\section{Methods}

\section{Data source}

This was a population-based cohort study. Data were obtained from the Longitudinal Health Insurance Database 2005, which was released by the Taiwan National Health Research Institute in 2006. The longitudinal database is a subset of the National Health Insurance Research Database and contains all medical claims data for one million beneficiaries randomly sampled from the 25.68 million enrollees of the National Health Insurance Program. The National Health Insurance Program, established in March 1995, is a system of universal health care under which beneficiaries can freely access all contracted medical facilities and institutions around the country for low copayments. As of 2007, up to $98 \%$ of Taiwan's population was enrolled in this program.

Data files in the National Health Insurance Research Database include all ambulatory claims, inpatient claims, details of ambulatory care and inpatient orders, and prescriptions dispensed at contracted pharmacies. The claims data are generally accurate; to date, more than 300 studies based on these data have been published in peer-reviewed journals. ${ }^{12,13}$

Because we used deidentified secondary data released to the public for research purposes, our study was exempt from full review by the Institutional Review Board after consultation with the Director of Taipei Medical University Institutional Review Board.

\section{Study population}

The study group of patients with COPD comprised all patients who had either received a diagnosis of COPD (codes 491, 492 and 496 of the International Classification of Diseases, 9th Revision, clinical modification [ICD-9-CM]) during two or more outpatient visits within 12 months or were admitted to hospital with a primary diagnosis of COPD between Jan. 1, 2004, and Dec. 31, 2005. Cohort entry was defined as the date when patients received their first eligible diagnosis. We included at entry only patients who were 50 years of age or older and had received respiratory medications for treatment of COPD, including inhaled and oral $\beta_{2}$-agonists, anticholinergic agents, corticosteroids and theophylline, for more than one month.

Each patient with COPD was matched by age (50-59, 60-69 or $\geq 70$ years), sex and year of cohort entry to four control patients from among the remaining patients in the database who had no diagnostic codes for COPD between 1996 and 2006. Because the National Health Insurance Program was started in 1995, we were able to track medical claims data only back to 1996 . For the comparison cohort, the first medical claim during the matched year was assigned as the date of cohort entry. Patients who had HIV infection or who had received a diagnosis of herpes zoster before the date of cohort entry were excluded from both the study and comparison cohorts. All patients were followed from the date of cohort entry until they developed herpes zoster or until Dec. 31, 2006, whichever was earliest.

\section{Covariables}

Medication exposure was identified from the drug prescription database. Patients were considered users of inhaled or systemic corticosteroids if they had been dispensed any form of inhaled or systemic steroids for more than 60 days during the follow-up period.

Sociodemographic characteristics, including age, sex, place of residence, urbanization level and monthly income, were obtained for all patients. We stratified all communities in Taiwan into five levels based on prior study, with level one referring to the most urbanized communities and level five referring to the least urbanized. ${ }^{14}$ Comorbid medical diseases that are well-known risk factors of herpes zoster, including malignancy (i.e., carcinoma, sarcoma, lymphoma and leukemia), rheumatic diseases (i.e., systemic lupus erythematosus, rheumatoid arthritis, Sjogren syndrome, scleroderma, dermatomyositis or polymyositis, and other connective tissue diseases) and diabetes, were identified from inpatient and outpatient diagnoses. ${ }^{4.5}$ Other medical conditions (e.g., myocardial infarction, congestive heart failure, peripheral vascular disease, cerebrovascular disease, dementia, peptic ulcer disease, liver disease, hemiplegia or paraplegia, and renal disease) were incorporated into a modified version of the Charlson index. Because COPD was the condition being evaluated, and malignancy, rheumatic diseases and diabetes were treated as individual covariates, the latter three were not included. Each condition was assigned a weight, and weightings were summed to provide a total score. ${ }^{15}$ 


\section{Statistical analysis}

We carried out $\chi^{2}$ tests to explore differences in sociodemographic characteristics and comorbidities between the COPD and comparison cohorts. The Kaplan-Meier method was used to estimate the cumulative hazard rate of herpes zoster, and a log-rank test was used to examine differences between the survival curves. Cox proportional hazard models were performed to compare the hazard ratio (HR) of herpes zoster between the COPD and comparison cohorts after stratification by age group. We adjusted for potential confounding factors in the multivariate analysis; including diabetes, cancer, rheumatic diseases, use of inhaled or oral corticosteroids, Charlson comorbidity index, monthly income and urbanization level of the community in which the patient resided. Potential first-order interactions between COPD and these variables were investigated, and no significant interaction was found.

We performed a further analysis to examine the risk of herpes zoster with and without use of steroids among patients with COPD. A multivariable Cox proportional model was conducted to investigate the HRs for development of herpes zoster for patients in the COPD cohort not using steroids, for those using inhaled corticosteroids only and for those using oral steroids compared with the comparison cohort. For all analyses, investigative diagnostic log-log survival plots were used to confirm that the proportional hazards assumption was met.

\section{Results}

We identified 8486 patients with a diagnosis of COPD who matched the inclusion criteria, and 33944 patients in the comparison cohort. The distributions of sociodemographic characteristics, comorbid medical disorders and steroid use for the COPD and comparison cohorts are shown in Table 1. Compared with patients without COPD, patients with COPD were more likely to have rheumatic disease, to use inhaled or oral steroids, to have higher Charlson comorbidity index and lower monthly incomes and to live in less urbanized areas of Taiwan.

Of the total sample of 42430 patients, $1080 \mathrm{had}$ incident herpes zoster during the follow-up period. There were 321 cases of herpes zoster identified in the COPD cohort, with an incidence rate of 16.4 per 1000 person-years, and 759 cases of herpes zoster in the comparison cohort, with an incidence rate of 8.8 per 1000 person-years. Cox regression analysis showed that the crude HR of herpes zoster was 1.98 times greater for patients with COPD (95\% confidence interval [CI] 1.73-2.26) than for the comparison cohort. The HR remained signifi-

Table 1: Demographic characteristics and comorbidities of patients with chronic obstructive pulmonary disease and patients in the comparison cohort $(n=42430)$

\begin{tabular}{|c|c|c|c|}
\hline Characteristic & $\begin{array}{c}\text { Patients with } \\
\text { COPD, no. }(\%) \\
n=8486\end{array}$ & $\begin{array}{c}\text { Comparison } \\
\text { patients, no. (\%) } \\
n=33944\end{array}$ & $p$ value* \\
\hline
\end{tabular}

\begin{tabular}{|lcrll|}
\hline Age, yr & & & \\
\hline $50-59$ & $1343(15.8)$ & $5372(15.8)$ & \\
\hline $60-69$ & $2321(27.4)$ & $9284(27.4)$ & \\
\hline$\geq 70$ & $4822(56.8)$ & $19288(56.8)$ & \\
\hline Sex & & & & \\
\hline Male & $5858(69.0)$ & $23432(69.0)$ & \\
\hline Female & $2628(31.0)$ & $10512(31.0)$ & \\
\hline Follow-up, mo, mean (SD) & $27.7(7.7)$ & $30.9(6.4)$ & $<0.001$ \\
\hline Monthly incomet & & & $<0.001$ \\
\hline
\end{tabular}

\begin{tabular}{|lrrr|}
\hline 0 & $2457(29.0)$ & $10472(30.9)$ \\
\hline NT\$1-15840 & $2442(28.8)$ & $8148(24.0)$ \\
\hline NT\$15 841-\$25000 & $3063(36.1)$ & $12391(36.5)$ \\
\hline$\geq$ NT\$25001 & $524(6.2)$ & $2933(8.6)$
\end{tabular}

\begin{tabular}{|cccc} 
Urbanization level & & 0.001 \\
\hline 1 & $1964(23.1)$ & $8878(26.2)$ &
\end{tabular}

\begin{tabular}{|c|c|c|c|}
\hline 1 & 1964 (23.1) & $8878(26.2)$ & \\
\hline 2 & $2213(26.1)$ & $8647(25.5)$ & \\
\hline 3 & $1241(14.6)$ & $5226(15.4)$ & \\
\hline 4 & $1510(17.8)$ & 5781 (17.0) & \\
\hline 5 & $1558(18.4)$ & $5412(18.4)$ & \\
\hline Geographic region & & & 0.022 \\
\hline Northern & 3644 (42.9) & $14654(43.2)$ & \\
\hline Central & 2115 (24.9) & $8313(24.5)$ & \\
\hline Southern & $2420(28.5)$ & $9950(29.3)$ & \\
\hline Eastern & $307 \quad(3.6)$ & $1027 \quad(3.0)$ & \\
\hline \multicolumn{4}{|l|}{ Comorbidity } \\
\hline Diabetes mellitus & & & 0.132 \\
\hline Yes & $1507(17.8)$ & 6268 (18.5) & \\
\hline No & $6979(82.2)$ & $27676(81.5)$ & \\
\hline Rheumatic disease & & & $<0.001$ \\
\hline Yes & $204 \quad(2.4)$ & $485 \quad(1.4)$ & \\
\hline No & $8282(97.6)$ & 33459 (98.6) & \\
\hline Cancer & & & 0.050 \\
\hline Yes & $503 \quad(5.9)$ & $1828 \quad(5.4)$ & \\
\hline No & $7983(94.1)$ & 32116 (94.6) & \\
\hline $\begin{array}{l}\text { User of inhaled } \\
\text { corticosteroids only }\end{array}$ & & & $<0.001$ \\
\hline Yes & $513 \quad(6.0)$ & $32(0.1)$ & \\
\hline No & $7973(94.0)$ & 33912 (99.9) & \\
\hline User of oral corticosteroids & & & $<0.001$ \\
\hline Yes & $1424(17.5)$ & $577 \quad(1.7)$ & \\
\hline No & 7062 (82.5) & 33367 (98.3) & \\
\hline $\begin{array}{l}\text { Charlson comorbidity index } \\
\text { score, mean }\end{array}$ & 1.24 & 0.77 & $<0.001$ \\
\hline \multicolumn{4}{|c|}{$\begin{array}{l}\text { Note: } \text { COPD }=\text { chronic obstructive pulmonary disease, NT = New Taiwan dollars, SD = standard } \\
\text { deviation. } \\
{ }^{*} p \text { values were calculated using Pearson } \chi^{2} \text { test. } \\
\text { †In New Taiwan Dollars, with an exchange rate in } 2005 \text { of } 1 \text { USD }=31.7 \text { NT. }\end{array}$} \\
\hline
\end{tabular}


cant after adjusting for potential confounders (adjusted HR 1.68, 95\% CI 1.45-1.95).

The results of the analysis, stratified by age of patients, are presented in Table 2. In all age categories, patients with COPD had a significantly higher risk of herpes zoster compared with those without COPD. The adjusted HRs for herpes zoster were 1.85 (95\% CI 1.27-2.70) in the 5059 age group, 1.65 (95\% CI 1.24-2.20) in the 60-69 age group and 1.68 (95\% CI 1.38-2.04) in the group aged 70 years or older.

The Kaplan-Meier survival curves for herpes zoster in the COPD and comparison groups over a three-year period are shown in Figure 1. The log-rank test showed a significant difference in cumulative event rates between these two groups $(p<0.001)$.

The results of further analysis, in which the COPD cohort was divided into three groups according to use of steroids, are shown in Table 3.
When compared with the comparison cohort, the adjusted HR of herpes zoster for patients with COPD not using steroids in any form was 1.67 (95\% CI 1.43-1.96). The adjusted HR of herpes zoster was slightly greater for patients with COPD using inhaled corticosteroids only (adjusted HR $2.09,95 \%$ CI 1.38-3.16) and was greatest for patients with COPD using oral steroids (adjusted HR 3.00, 95\% CI 2.40-3.75).

\section{Interpretation}

Our cohort study showed that, after controlling for other herpes zoster risk factors, patients with COPD are at increased risk for herpes zoster compared with the general population. The risk of herpes zoster associated with COPD is greater among patients who use inhaled or oral corticosteroids therapy than among patients without corticosteroids therapy.

Table 2: Crude and adjusted hazard ratios for herpes zoster during the follow-up period for patients with chronic obstructive pulmonary disease and patients in the comparison group, stratified by age

\begin{tabular}{|c|c|c|c|c|c|}
\hline Age, yr & $\begin{array}{l}\text { Had herpes } \\
\text { zoster }\end{array}$ & $\begin{array}{l}\text { Person-years } \\
\text { at risk }\end{array}$ & $\begin{array}{c}\text { Incidence per } \\
1000 \text { person-years }\end{array}$ & $\begin{array}{l}\text { Crude HR } \\
(95 \% \mathrm{Cl})\end{array}$ & $\begin{array}{l}\text { Adjusted HR* } \\
\qquad(95 \% \mathrm{Cl})\end{array}$ \\
\hline \multicolumn{6}{|l|}{$50-59$} \\
\hline COPD & 44 & 2941.47 & 14.26 & $1.81(1.27-2.56)$ & $1.85(1.27-2.70)$ \\
\hline Comparison & 112 & 13089.16 & 8.56 & 1.00 & 1.00 \\
\hline \multicolumn{6}{|l|}{$60-69$} \\
\hline COPD & 84 & 5269.45 & 15.94 & $1.77(1.38-2.28)$ & $1.65(1.24-2.20)$ \\
\hline Comparison & 220 & 23540.40 & 9.35 & 1.00 & 1.00 \\
\hline \multicolumn{6}{|l|}{$\geq 70$} \\
\hline COPD & 193 & 11073.02 & 17.43 & $2.10(1.77-2.49)$ & $1.68(1.38-2.04)$ \\
\hline Comparison & 427 & 49668.80 & 8.60 & 1.00 & 1.00 \\
\hline
\end{tabular}

Table 3: Crude and adjusted hazard ratios for herpes zoster for patients without steroid therapy, with inhaled corticosteroid therapy only and with oral steroid therapy, compared to control patients

\begin{tabular}{|lccccc|}
\hline Patient group & $\begin{array}{c}\text { Had herpes } \\
\text { zoster }\end{array}$ & $\begin{array}{c}\text { Person-years } \\
\text { at risk }\end{array}$ & $\begin{array}{c}\text { Incidence per } \\
1000 \text { person-years }\end{array}$ & $\begin{array}{c}\text { Crude HR } \\
(95 \% \mathrm{Cl})\end{array}$ & $\begin{array}{c}\text { Adjusted HR* } \\
(95 \% \mathrm{Cl})\end{array}$ \\
\hline Comparison & 759 & 86298.37 & 8.80 & 1.00 & 1.00 \\
\hline $\begin{array}{l}\text { Patients with COPD } \\
\begin{array}{l}\text { No use of } \\
\text { corticosteroids }\end{array}\end{array}$ & 209 & 14643.74 & 14.27 & $1.69(1.45-1.97)$ & $1.67(1.43-1.96)$ \\
\hline $\begin{array}{l}\text { Inhaled } \\
\text { corticosteroids }\end{array}$ & 23 & 1251.90 & 18.37 & $2.11(1.39-3.19)$ & $2.09(1.38-3.16)$ \\
\hline $\begin{array}{l}\text { Oral } \\
\text { corticosteroids }\end{array}$ & 89 & 3388.80 & 26.26 & $3.03(2.43-3.77)$ & $3.00(2.40-3.75)$ \\
\hline
\end{tabular}

Note: $\mathrm{Cl}=$ confidence interval, $\mathrm{COPD}=$ chronic obstructive pulmonary disease, $\mathrm{HR}=$ hazard ratio.

*Adjusted for sex, age (as a continuous variable), diabetes mellitus, rheumatic diseases, cancer, Charlson comorbidity index score, use of corticosteroids, monthly income, geographic region and urbanization level. 
In a recent retrospective study comparing 355 patients with COPD and 44 control patients, Patel and colleagues ${ }^{16}$ found that self-reported history of herpes zoster was more common among patients with COPD than among control patients. However, a relatively small study sample and recall bias were major limitations of their study. Susceptibility to respiratory tract infections among patients with COPD has frequently been reported in the literature.? Our results suggest that patients may also be predisposed to development of herpes zoster by chronic inflammation and immune dysregulation in COPD. Systemic immune disturbances have been observed in patients with COPD. ${ }^{17-19}$ For example, Prieto and colleagues ${ }^{17}$ found that cytotoxic activity of peripheral blood natural killer cells and phagocytic activity of peripheral blood monocytes, macrophages and polymorphonuclear cells were significantly reduced in patients with COPD compared with healthy control patients. Systemic cell-mediated immunity has been found to be impaired in COPD and to be associated with acute respiratory tract infection among patients with COPD. ${ }^{18}$ Further, recent data have shown that COPD and aging produce significant molecular similarities in immunologic alterations. ${ }^{19}$ Immunosenescence in aging is a well-known risk factor for herpes zoster; 5 immune system changes in COPD may further render such patients more vulnerable to varicella-zoster virus reactivation than age-matched control patients. We found that risk for herpes zoster was increased among patients with COPD in all age groups. It is possible that the altered immunity in COPD causes similar effects in terms of reactivating varicellazoster virus across different age groups.

The slightly higher HR of herpes zoster in patients with COPD using inhaled corticosteroids and the highest HR of herpes zoster among those using oral steroids may be related to the immunosuppressive effect of corticosteroids. Systemic steroids affect cellular immunity by inhibiting synthesis of inflammatory cytokines, reducing function of antigen-presenting cells, and suppressing $\mathrm{T}$ cell activation. ${ }^{20,21}$ Steroid-related immunosuppression can increase susceptibility to opportunistic and intracellular infections, such as herpes zoster. $^{22}$ Although oral corticosteroids have frequently been reported to be associated with the occurrence of herpes zoster, the link between inhaled corticosteroids and varicella-zoster virus reactivation is unclear. ${ }^{4.5}$ Although recent evidence suggests that inhaled corticosteroids suppress local immunity and increase the risk of pneumonia and pulmonary tuberculosis among patients with COPD, ${ }^{23,24}$ the impact of inhaled corticosteroids on systemic immunity remains controversial. ${ }^{25}$ Abzug and Cotton ${ }^{26}$ have reported severe varicella infection after use of intranasal corticosteroids, and Choong and colleagues ${ }^{27}$ reported severe varicella infection after use of inhaled corticosteroids. Patel and colleagues also observed a higher rate of self-reported herpes zoster among patients with COPD using inhaled corticosteroids compared with those not using inhaled corticosteroids ( $15.1 \%$ v. $7.2 \%)$; however, the difference was not significant. ${ }^{16}$ Our study suggests that inhaled corticosteroids may have potential for systemic absorption and may impair cellular immunity, but their immunosuppressive effect is not as great as that of systemic steroids.

Another possible explanation for our observation that the risk of herpes zoster was greater among patients using inhaled or oral corticosteroids relative to the general population is that the use of these medications often indicates more severe or unstable disease. It is possible that increased disease severity further contributed to the increased risk of herpes zoster associated with COPD.

\section{Limitations}

Our study has limitations. First, because almost all herpes zoster cases were diagnosed clinically without serologic or virologic confirmation, some non-

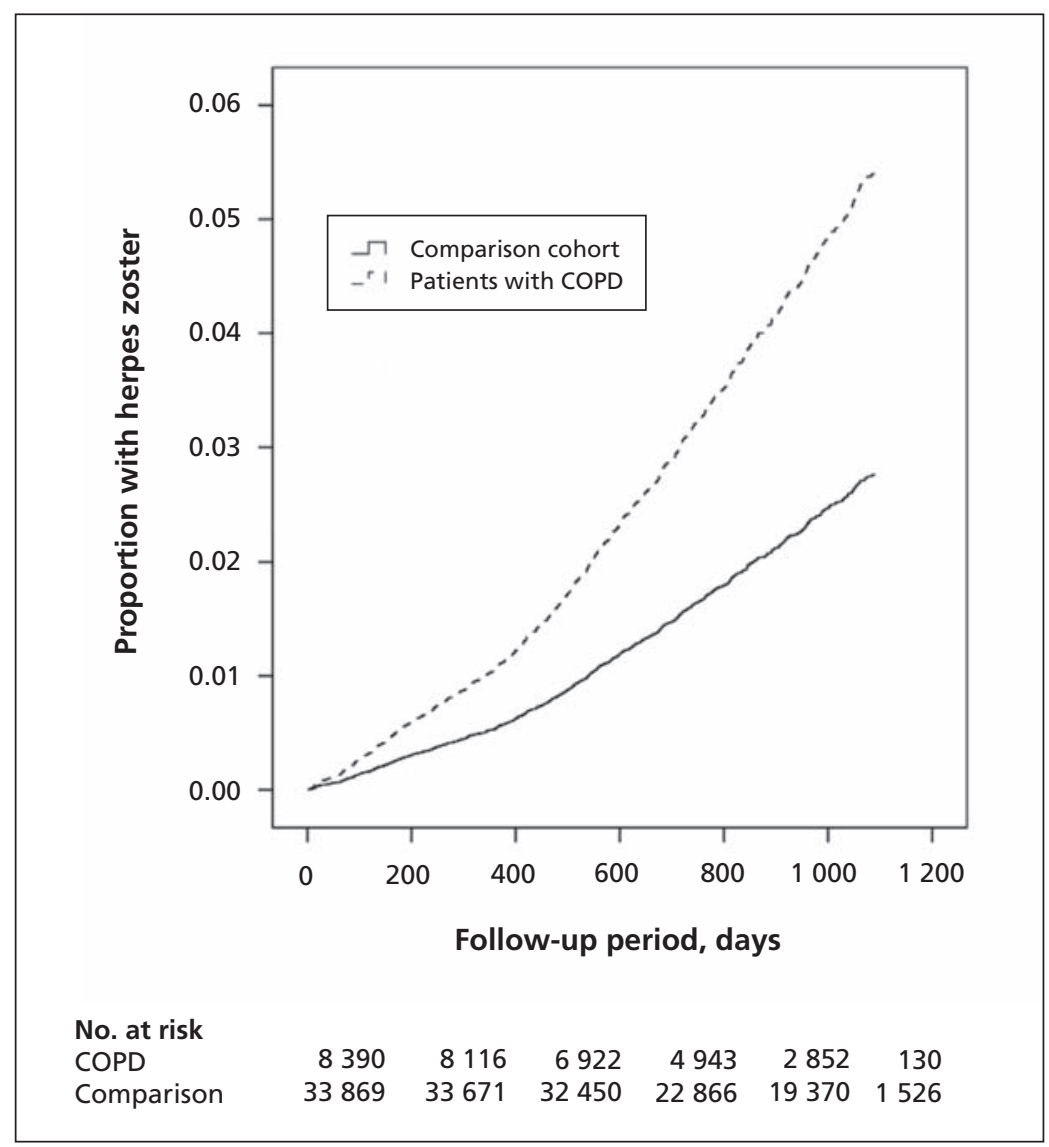

Figure 1: Herpes zoster event rates for patients with chronic obstructive pulmonary disease and comparison patients from 2004 to 2006. Log-rank test: $p<0.001$ 
zoster cases (e.g., herpes simplex) might have been misdiagnosed as herpes zoster. ${ }^{28}$ Second, information about cigarette smoking and alcohol consumption was lacking in the administrative database; therefore, we could not adjust for effects of smoking and drinking on the risk of herpes zoster among patients with COPD, which may have left residual confounding. Third, data on death could not be obtained in the analysis. Given that mortality among study patients with COPD would be higher than in the comparison cohort; ${ }^{29}$ it is possible that patients who did not become ill with herpes zoster attack and who died during the study period would be assumed alive and followed until the end of the study. This differential misclassification may have biased our results toward the null hypothesis. Finally, data indicating severity of COPD, such as forced expiratory volume in one second as a percentage of predicted normal values $\left(\mathrm{FEV}_{1} \%\right.$ predicted), were not available in our study.

\section{Conclusion}

Our results suggest that COPD, like other inflammatory diseases (e.g., rheumatoid arthritis, inflammatory bowel disease) was associated with an increased risk of herpes zoster relative to that in the general population. The HR of herpes zoster was slightly greater for patients with COPD using inhaled corticosteroids and was greatest for patients with COPD using oral steroids. To prevent the substantial morbidity ensuing from this vexing disease, it is recommended that patients with COPD receive zoster vaccination ${ }^{30}$ in addition to influenza and pneumococcal immunization.

\section{References}

1. Straus SE, Oxman MN, Schmader KE. Varicella and herpes zoster. In: Wolff K, Goldsmith LA, Katz SI, et al. editors. Fitzpatrick's dermatology in general medicine. New York (NY): McGraw-Hill Companies, Inc; 2008. p. 1885-98.

2. Lydick E, Epstein RS, Himmelberger D, et al. Herpes zoster and quality of life: a self-limited disease with severe impact. Neurology 1995;45:S52-3.

3. Johnson RW, Bouhassira D, Kassianos G, et al. The impact of herpes zoster and post-herpetic neuralgia on quality-of-life. BMC Med 2010;8:37.

4. Smitten AL, Choi HK, Hochberg MC, et al. The risk of herpes zoster in patients with rheumatoid arthritis in the United States and the United Kingdom. Arthritis Rheum 2007;57:1431-8.

5. Thomas SL, Hall AJ. What does epidemiology tell us about risk factors for herpes zoster? Lancet Infect Dis 2004;4:26-33.

6. Cosio MG, Saetta M, Agusti A. Immunologic aspects of chronic obstructive pulmonary disease. $N$ Engl J Med 2009;360:2445-54.

7. Sethi S. Bacterial infection and the pathogenesis of COPD Chest 2000;117:286S-91S

8. Vestbo J, Hogg JC. Convergence of the epidemiology and pathology of COPD. Thorax 2006;61:86-8.

9. Wolfe F, Michaud K, Chakravarty EF. Rates and predictors of herpes zoster in patients with rheumatoid arthritis and noninflammatory musculoskeletal disorders. Rheumatology (Oxford) 2006; $45: 1370-5$

10. Gupta G, Lautenbach E, Lewis JD. Incidence and risk factors for herpes zoster among patients with inflammatory bowel disease. Clin Gastroenterol Hepatol 2006;4:1483-90.

11. Hambleton S, Gershon AA. Preventing varicella-zoster disease. Clin Microbiol Rev 2005;18:70-80.

12. About NHI. Taipei (Taiwan): Bureau of National Health Insurance;
2010. Available: www.nhi.gov.tw/english/webdata.asp?menu=11 \&menu_id=290\&webdata_id=2974 (accessed 2010 Feb. 4).

13. Chen YC, Yeh, HY, et al. Taiwan's National Health Insurance Database: administrative health care database as study object in biblometrics. Scientometrics 2011;86:365-80

14. Chung KH, Lee HC, Kao S, et al. Urbanicity and methods of suicide: a nationwide population-based study. J Urban Health 2008;85:136-45

15. Souza RC, Pinheiro RS, Coeli CM, et al. The Charlson comorbidity index $(\mathrm{CCI})$ for adjustment of hip fracture mortality in the elderly: analysis of the importance of recording secondary diagnoses. Cad Saude Publica 2008;24:315-22.

16. Patel ARC, Karmali S, Donaldson G. Prevalence of herpes zoster in COPD and relationships with inhaled corticosteroids, exacerbation frequency and FEV1. Am J Respir Crit Care Med 2010;181:A5926.

17. Prieto A, Reyes E, Bernstein ED, et al. Defective natural killer and phagocytic activities in chronic obstructive pulmonary disease are restored by glycophosphopeptical (inmunoferon). Am J Respir Crit Care Med 2001;163:1578-83.

18. Takabatake N, Sata M, Abe S, et al. Impaired systemic cell-mediated immunity and increased susceptibility to acute respiratory tract infections in patients with COPD. Respir Med 2005;99:485-92.

19. Sharma G, Hanania NA, Shim YM. The aging immune system and its relationship to the development of chronic obstructive pulmonary disease. Proc Am Thorac Soc 2009;6:573-80.

20. Cupps TR, Fauci AS. Corticosteroid-mediated immunoregulation in man. Immunol Rev 1982;65:133-55.

21. Holt PG, Thomas JA. Steroids inhibit uptake and/or processing but not presentation of antigen by airway dendritic cells. Immunology 1997;91:145-50.

22. Mukwaya G. Immunosuppressive effects and infections associated with corticosteroid therapy. Pediatr Infect Dis J 1988;7:499-504.

23. Drummond MB, Dasenbrook EC, Pitz MW, et al. Inhaled corticosteroids in patients with stable chronic obstructive pulmonary disease: a systematic review and meta-analysis. JAMA 2008; 300:2407-16.

24. Shu CC, Wu HD, Yu MC, et al. Use of high-dose inhaled corticosteroids is associated with pulmonary tuberculosis in patients with chronic obstructive pulmonary disease. Medicine (Baltimore) 2010;89:53-61.

25. Levy J, Zalkinder I, Kuperman O, et al. Effect of prolonged use of inhaled steroids on the cellular immunity of children with asthma. J Allergy Clin Immunol 1995;95:806-12.

26. Abzug MJ, Cotton MF. Severe chickenpox after intranasal use of corticosteroids. J Pediatr 1993;123:577-9.

27. Choong K, Zwaigenbaum L, Onyett H. Severe varicella after low dose inhaled corticosteroids. Pediatr Infect Dis J 1995;14:809-11.

28. Kalman CM, Laskin OL. Herpes zoster and zosteriform herpes simplex virus infections in immunocompetent adults. Am J Med 1986;81:775-8

29. Lopez AD, Shibuya K, Rao C, et al. Chronic obstructive pulmonary disease: current burden and future projections. Eur Respir J 2006;27:397-412.

30. Oxman MN, Levin MJ, Johnson GR, et al. A vaccine to prevent herpes zoster and postherpetic neuralgia in older adults. $N$ Engl J Med 2005;352:2271-84.

Affiliations: From the Department of Dermatology (Yang, K-H Wang), School of Medicine, College of Medicine, Taipei Medical University; the Department of Dermatology (Yang, K-H Wang), Taipei Medical University Hospital; the School of Public Health (Chen), College of Public Health and Nutrition, Taipei Medical University; the Department of Internal Medicine (C-Y Wang), Cardinal Tien Hospital, Fu-Jen Catholic University; and the Biostatistics and Research Consultation Center (Lin), Taipei Medical University, Taipei, Taiwan

Contributors: Ya-Wen Yang designed the study and interpreted the results of data analysis. Yi-Hua Chen, Kuo-Hsien Wang and Chen-Yi Wang interpreted the study results. HuiWen Lin designed the study and analyzed the data. All of the authors were involved in writing the manuscript, and all of them critically revised it for important intellectual content and approved the final version submitted for publication.

Funding: This study was supported by a grant (no. 99TMUTMUH-11) from the Taipei Medical University Hospital.

Acknowledgement: This study is based in part on data from the National Health Insurance Research Database provided by the Bureau of National Health Insurance, Department of Health and managed by National Health Research Institutes in Taiwan. 